\title{
K- and L-band spectroscopy of Be stars
}

\author{
Anahí Granada ${ }^{1,2,4}$, María L. Arias ${ }^{1,2}$, Lydia S. Cidale $^{1,2}$ \\ and Ronald E. Mennickent ${ }^{3}$ \\ ${ }^{1}$ Facultad de Ciencias Astronómicas y Geofísicas, Universidad Nacional de La Plata, Argentina \\ ${ }^{2}$ Instituto de Astrofísica La Plata, CCT La Plata-CONICET-UNLP, Argentina \\ ${ }^{3}$ Departamento de Astronomía, Universidad de Concepción, Chile \\ ${ }^{4}$ Observatoire de Genève. Université de Genève, Suisse
}

email: granada@f caglp.unlp.edu.ar

\begin{abstract}
We describe the behaviour of IR hydrogen emission lines of a sample of Be stars and discuss the physical properties of the circumstellar envelopes of Be stars classified in Groups I and II (Mennickent et al. 2009). We find that while Humphreys and Pfund lines of Group I stars form in an optically thick envelope/disk, Group II stars show Pfund lines that form in an optically thick medium and Humphreys lines originating in optically thinner regions. The transition between Groups I and II could be understood in terms of the evolution of the circumstellar disk of the star and might bring clues on the mechanism originating the Be phenomenon.
\end{abstract}

Keywords. stars: emission-line, Be, circumstellar matter

\section{Introduction}

Be stars have a circumstellar envelope revealed in the infrared through moderate flux excesses and the appearance of hydrogen recombination lines. K- and L- band spectra of Be stars present numerous lines of Pfund (Pf), Humphreys $(\mathrm{Hu})$ and Brackett $(\mathrm{Br})$ series. Their profiles are sensitive to the physical properties and dynamical structure of the line-forming regions and thus become useful probes of circumstellar environments. An inspection of the L-band hydrogen spectra of Be stars allows a classification in three groups following Mennickent et al. (2009): Group I contains the stars with Br $\alpha$ and Pf $\gamma$ equally intense as $\mathrm{Hu}$ lines, Group II consists of those with $\operatorname{Br} \alpha$ and $\operatorname{Pf} \gamma$ more intense than $\mathrm{Hu}$ lines, and Group III is made up of those with no detected emission. This classification scheme reflects the optical depth conditions in the Be star envelope. Group I stars have a more compact $\mathrm{Hu}$ line-forming region than Group II stars, whereas Group III stars might have lost their envelopes (Mennickent et al. 2009, Granada et al. 2010). In this work we analize K- and L- band spectra of a sample of 26 B-type emission line stars (see Table 1) and relate them to the optical depths of their line-forming regions.

\section{Results}

We show the line fluxes of $\mathrm{Hu}$ and $\mathrm{Pf}$ lines relative to a reference transition (Fig. 1), for the stars in Table 1. The continous lines indicate the optically thin limit predicted by Menzel Case B recombination theory while the dashed line represents the thick case.

$\mathrm{Hu}$ and Pf lines of Group I stars come from an optically thick envelope (Figs. 1.1a and 1.1b). Fig. 1.2a shows the range of $\mathrm{Hu}$ line flux ratios covered by Be stars of Group II. In some cases these ratios are close to Menzel Case B, whereas some objects depart from this case. Pf line ratios (Fig. 1.2b) correspond to an optically thick envelope. B[e] stars have $\mathrm{Hu}$ and $\mathrm{Pf}$ line ratios (Fig. 1.3), close to those of Menzel Case B, likely to form in an isothermal stellar wind (Lenorzer et al. 2002a). 
Table 1. Sample. a)Lenorzer et al.(2002b); b)Mennickent et al.(2009); c)Granada et al.(2010)

\begin{tabular}{|c|c|c|c|c|c|c|c|c|c|}
\hline Star (HD) & Name & S.T. & Group & Ref $\mid$ & Star (HD) & Name & S.T. & Group & Ref \\
\hline & MWC349A & O9III $[\mathrm{e}]$ & II & $\mathrm{a}$ & 120991 & V767 Cen & B3IIIe & I & b \\
\hline 5394 & $\gamma \mathrm{Cas}$ & B0IVesh & II & $\mathrm{a}$ & 148259 & OZ Nor & B2II & I & b \\
\hline 20336 & BK Cam & $\mathrm{B} 2.5 \mathrm{Ve}$ & I & $\mathrm{c}$ & 162732 & 88 Her & Bpshe & I & $\mathrm{c}$ \\
\hline 23862 & $28 \mathrm{Tau}$ & B8 IVev & II & c & 164284 & $66 \mathrm{Oph}$ & $\mathrm{B} 2 \mathrm{Ve}$ & II & c \\
\hline 29441 & V1150 Tau & $\mathrm{B} 2.5 \mathrm{Vne}$ & I & $\mathrm{b}$ & 178175 & V $4024 \mathrm{Sgr}$ & $\mathrm{B} 2 \mathrm{~V}$ & I & $\mathrm{b}$ \\
\hline 45677 & MWC 142 & $\mathrm{~B} 2 \mathrm{~V}[\mathrm{e}]$ & II & $\mathrm{a}$ & 183656 & V923 Aql & Bpshe & II & $\mathrm{c}$ \\
\hline 50013 & $\kappa \mathrm{CMa}$ & B1.5IVne & I & a & 186272 & V341 Sge & $\mathrm{B} 2.5 \mathrm{~V}$ & II & $\mathrm{b}$ \\
\hline 56139 & $\omega \mathrm{CMa}$ & $\mathrm{B} 2 \mathrm{IV}-\mathrm{Ve}$ & II & $\mathrm{a}$ & 187811 & $12 \mathrm{Vul}$ & $\mathrm{B} 2.5 \mathrm{Ve}$ & I,II & $a, b$ \\
\hline 93308 & $\eta$ Car & Bpe & II & a & 191610 & $28 \mathrm{Cyg}$ & $\mathrm{B} 2.5 \mathrm{Ve}$ & II & $\mathrm{a}, \mathrm{c}$ \\
\hline 94910 & $\mathrm{AG} \mathrm{Car}$ & B2pe & II & $\mathrm{a}$ & 193237 & P Cyg & B2pe & II & $\mathrm{a}$ \\
\hline 105521 & V817 Cen & B3 IVe & I & $\mathrm{b}$ & 200775 & MWC 361 & $\mathrm{~B} 2 \mathrm{~V}[\mathrm{e}]$ & II & $\mathrm{a}$ \\
\hline 105435 & $\delta$ Cen & B2IVne & II & $\mathrm{b}$ & 209409 & omi Agr & B7IVe & II & a \\
\hline 120324 & $\mu \mathrm{Cen}$ & B2IV-Ve & II & $\mathrm{b}$ & 217050 & EW Lac & B3 IVshe & II & $\mathrm{c}$ \\
\hline
\end{tabular}
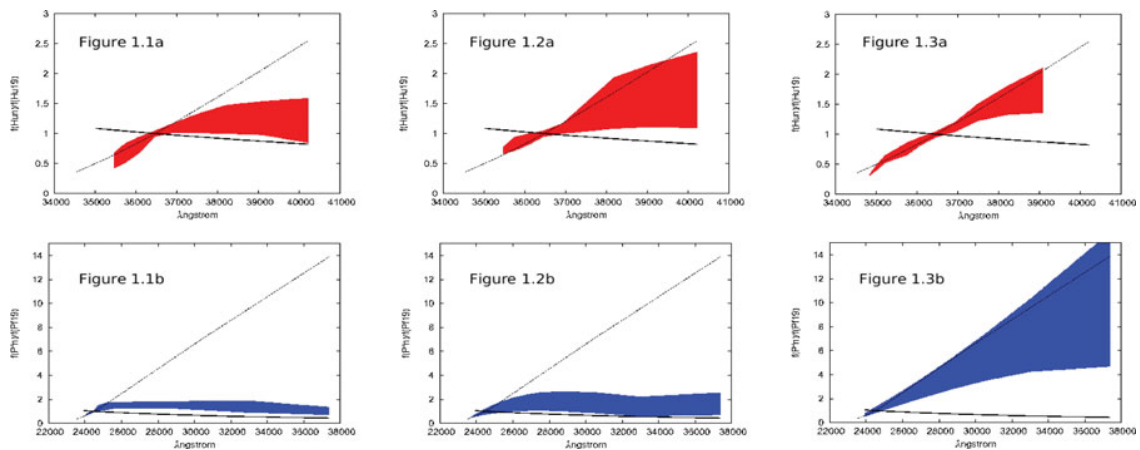

Figure 1. Line Flux Ratios: 1) Group I; 2) Group II; 3) Other emission lines

\section{Discussion}

Most of the objects in Table 1 are B2-3 type stars. Thus, if we consider similar central stars, the differences observed in the IR spectra evidence differences in the line-forming regions. We obtain a rough estimate of the column density of atoms in the lower excitation level (that is $\mathrm{N}_{6}$ for $\mathrm{Hu}$ lines and $\mathrm{N}_{5}$ for Pf lines) as well as the relative extension, $\Delta \mathrm{r}$ of the forming region of $\mathrm{Hu}\left(\mathrm{R}_{H u n} / \mathrm{R}_{H u 16}\right)$ and $\mathrm{Pf}\left(\mathrm{R}_{P f n} / \mathrm{R}_{P f 16}\right)$ lines, using line flux ratios (Granada et al. 2010). We find that for Group I stars the $\mathrm{Hu}$ line-forming regions seem to be more dense and compact than those for Group II stars; whereas the mean column density obtained for Group I stars ranges from 1.2 to $3.7 \times 10^{14} \mathrm{~cm}^{-1}$ and $\Delta \mathrm{r}$ from 0.38 to 1.28 , for $\mathrm{Hu}_{18}$ to $\mathrm{Hu}_{14}$, Group II stars have column densities from 0.22 to $1.39 \times 10^{14} \mathrm{~cm}^{-1}$ with $\Delta \mathrm{r}$ from 0.28 to 1.90 for the same lines. Moreover, higher members of Pf series for B2 stars of both Groups also form in compact and dense regions.

Many Be stars of our sample show strong spectroscopic variability. For $12 \mathrm{Vul}$ and 28 Cyg, we reported changes from one Group to another, which are possibly indicating structural changes in the circumstellar environment (Mennickent et al. 2009, Granada et al. 2010). Time resolved near-IR spectroscopy of Be stars would allow us to study the origin and evolution of the envelopes as well as to set constraints to different models.

\section{References}

Granada, A., Arias, M. L. \& Cidale, L. S. 2010, AJ, 139, 1983

Lenorzer, A., de Koter, A. \& Waters, L. B. F. M. 2002a, A\&A, 386, L5

Lenorzer, A., Vandenbussche, B., Morris, P., de Koter, A. et al. 2002b, A\&A A, 384, 473

Mennickent, R. E., Sabogal, B., Granada, A., \& Cidale, L. 2009, PASP, 121, 125 